\title{
THE SPITZER c2d SURVEY OF LARGE, NEARBY, INTERSTELLAR CLOUDS. VIII. SERPENS OBSERVED WITH MIPS
}

\author{
Paul M. Harvey, ${ }^{1}$ Luisa M. Rebull, ${ }^{2}$ Tim Brooke, ${ }^{3}$ William J. Spiesman, ${ }^{1}$ Nicholas Chapman, ${ }^{4}$ Tracy L. Huard, ${ }^{5}$ \\ Neal J. Evans II, ${ }^{1}$ Lucas Cieza, ${ }^{1}$ Shih-Ping Lai, ${ }^{4}$ Lori E. Allen,${ }^{5}$ Lee G. Mundy, ${ }^{4}$ Deborah L. Padgett, ${ }^{2}$ \\ Anneila I. Sargent, ${ }^{3}$ Karl R. Stapelfeldt, ${ }^{6}$ Philip C. Myers, ${ }^{5}$ Ewine F. van Dishoeck, ${ }^{7}$ \\ Geoffrey A. Blake, ${ }^{8}$ and David W. Koerner ${ }^{9}$ \\ Received 2007 February 3; accepted 2007 March 16
}

\begin{abstract}
We present maps of $1.5 \mathrm{deg}^{2}$ of the Serpens dark cloud at 24, 70, and $160 \mu \mathrm{m}$ observed with the Spitzer Space Telescope MIPS camera. We describe the observations and briefly discuss the data processing carried out by the $\mathrm{c} 2 \mathrm{~d}$ team on these data. More than 2400 compact sources have been extracted at $24 \mu \mathrm{m}$, nearly 100 at $70 \mu \mathrm{m}$, and four at $160 \mu \mathrm{m}$. We estimate completeness limits for our $24 \mu \mathrm{m}$ survey from Monte Carlo tests with artificial sources inserted into the Spitzer maps. We compare source counts, colors, and magnitudes in the Serpens cloud to two reference data sets: a $0.50 \mathrm{deg}^{2}$ set on a low-extinction region near the dark cloud, and a $5.3 \mathrm{deg}^{2}$ subset of the SWIRE ELAIS N1 data that was processed through our pipeline. These results show that there is an easily identifiable population of young stellar object candidates in the Serpens cloud that is not present in either of the reference data sets. We also show a comparison of visual extinction and cool dust emission illustrating a close correlation between the two and find that the most embedded YSO candidates are located in the areas of highest visual extinction.
\end{abstract}

Subject headings: infrared: general

\section{INTRODUCTION}

The Spitzer Space Telescope Legacy project "From Molecular Cores to Planet-forming Disks" includes IRAC and MIPS mapping of five large star-forming clouds (Evans et al. 2003). The Serpens cloud covers more than $10 \mathrm{deg}^{2}$ as mapped by optical extinction (Cambrésy 1999), but for reasons of practicality the $\mathrm{c} 2 \mathrm{~d}$ project was only able to observe $1.5 \mathrm{deg}^{2}$ with the MIPS instrument on Spitzer (further Spitzer observations of a larger area of Serpens are planned as part of an extended survey of the Gould Belt; L. E. Allen 2007, in preparation). At an assumed distance of $260 \mathrm{pc}$ (Straizys et al. 1996), the area mapped by c2d corresponds to $\sim 4.5 \times 7 \mathrm{pc}$. This paper is one in a series describing the IRAC and MIPS observations of each of the $\mathrm{c} 2 \mathrm{~d}$ clouds. Previous papers include those on IRAC observations of Serpens (Harvey et al. 2006), Chamaeleon (Porras et al. 2007), and Perseus (Jorgensen et al. 2006), as well as MIPS observations of Chamaeleon (Young et al. 2005), Perseus (Rebull et al. 2007), Lupus (Chapman et al. 2007), and Ophiuchus (Padgett et al. 2007).

Our observations of Serpens cover an area that includes the well-studied "core" cluster region, cluster A, together with the

\footnotetext{
1 Astronomy Department, University of Texas at Austin, Austin, TX 787120259; pmh@astro.as.utexas.edu,nje@astro.as.utexas.edu, lcieza@astro.as.utexas .edu, spies@astro.as.utexas.edu.

2 Spitzer Science Center, Pasadena, CA 91125; rebull@ipac.caltech.edu, dlp@ipac.caltech.edu.

3 Division of Physics, Mathematics, and Astronomy, California Institute of Technology, Pasadena, CA 91125; tyb@astro.caltech.edu, afs@astro.caltech.edu.

4 Astronomy Department, University of Maryland, College Park, MD 20742; chapman@astro.umd.edu, slai@astro.umd.edu,lgm@astro.umd.edu.

5 Smithsonian Astrophysical Observatory, Cambridge, MA 02138; leallen@ cfa.harvard.edu, thuard@cfa.harvard.edu,pmyers@cfa.harvard.edu.

6 Jet Propulsion Laboratory, California Institute of Technology, Pasadena, CA 91109; krs@exoplanet.jpl.nasa.gov.

7 Leiden Observatory, 2300 RA Leiden, Netherlands; ewine@strw.leidenuniv .nl. ${ }_{8}$

${ }^{8}$ Division of Geological and Planetary Sciences, California Institute of Technology, Pasadena, CA 91125; gab@gps.caltech.edu.

9 Department of Physics and Astronomy, Northern Arizona University, Flagstaff, AZ 86011-6010; koerner@physics.nau.edu.
}

newly discovered cluster B (Harvey et al. 2006; Djupvik et al. 2006) to the south, as well as the Herbig Ae/Be star, VV Ser. Significant portions of this cloud have been studied by previous space infrared missions, including IRAS (Zhang et al. 1988a, 1988b) and the Infrared Space Observatory (ISO; Kaas et al. 2004; Djupvik et al. 2006). The much higher sensitivity and longer wavelength capability of the Spitzer MIPS instrument, however, allow us both to detect very low luminosity infrared excess objects and to map very cool diffuse dust emission in the region. Our results are also complementary to the $1.1 \mathrm{~mm}$ mapping of the same region by Enoch et al. (2006). The combined results on Serpens using both the MIPS and IRAC observations are discussed in a companion paper, where we also give detailed object lists (Harvey et al. 2007).

In $\oint 2$ we describe details of the observations obtained from the MIPS instrument for Serpens and the data processing pipeline used to reduce the observations. In $\S 3$ we describe a number of results from our MIPS observations and correlations between them and the Two Micron All Sky Survey (2MASS) catalog (Skrutskie et al. 2006). We show in $\S 3.1$ that there is an excellent correlation between the coolest dust that we can observe, which emits at $160 \mu \mathrm{m}$, and the optical extinction in Serpens. We investigate the possibility of time variability at $24 \mu \mathrm{m}$ in our twoepoch data set in $\S 3.2$. In $\S 3.3$ we discuss our results statistically in terms of source counts and compare these to predictions of models of the Galaxy, as well as to the counts in the reference fields. We present color-color and color-magnitude plots of the population of infrared sources in $\S 3.4$ and discuss the separation of likely cloud members from the extensive background population of stars and extragalactic objects. In the final part of $\S 3$ we briefly describe some details of individual sources of particular interest.

\section{OBSERVATIONS AND DATA REDUCTION}

The MIPS observations cover an area where $A_{v}>6$ in the contour maps of Cambrésy (1999). In addition, a nearby offcloud region of $0.5 \mathrm{deg}^{2}$ was mapped for comparison with the 
TABLE 1

Summary of Observations

\begin{tabular}{ccccc}
\hline \hline Region & AOR & $\begin{array}{c}\text { Date } \\
(\mathrm{UT})\end{array}$ & $\begin{array}{c}l^{\mathrm{a}} \\
(\mathrm{deg})\end{array}$ & $\begin{array}{c}b^{\mathrm{a}} \\
(\mathrm{deg})\end{array}$ \\
\hline Serpens ..................... & 5713408 & 2004 Apr 05 23:40 & 31.5 & 5.4 \\
& 5713920 & 2004 Apr 06 04:05 & 31.5 & 5.3 \\
& 5713664 & 2004 Apr 06 00:22 & 31.6 & 5.2 \\
Off-cloud …............... & 5714176 & 2004 Apr 06 04:48 & 30.6 & 5.1 \\
& 5716736 & 2004 Apr 06 01:26 & 35.2 & 4.4 \\
& 5716992 & 2004 Apr 06 05:52 & 35.2 & 4.3 \\
\hline
\end{tabular}

${ }^{\text {a }}$ Coordinates $l$ and $b$ are listed for the center of the $24 \mu \mathrm{m}$ AOR.

cloud region. A summary of the regions observed is listed in Table 1 with the AOR (Astronomical Observation Request) number to facilitate access from the Spitzer archive. The regions covered at $24 \mu \mathrm{m}$ are outlined in Figure 1 against the $25 \mu \mathrm{m}$ IRAS sky. The observing strategy and basic MIPS data analysis for the c2d star-forming clouds have been described in detail by Rebull et al. (2007), but we summarize here the most important details. Fast scan maps were obtained at two separate epochs with a spacing between adjacent scan legs of $240^{\prime \prime}$ in each epoch. The second-epoch observations were offset by $125^{\prime \prime}$ from the first in the cross scan direction to fill in the $70 \mu \mathrm{m}$ sky coverage that would otherwise have been missed due to detector problems. The second-epoch scan was also offset $80^{\prime \prime}$ from the first in the scan direction to minimize missing $160 \mu \mathrm{m}$ data. For some of the c2d clouds, these offsets together with sky rotation were sufficient to give essentially complete one-epoch coverage at $160 \mu \mathrm{m}$, but for Serpens there were still small gaps between every two scan lines. Table 2 lists the sky coverage at each wavelength. The two observation epochs were separated in time by $\sim 6 \mathrm{hr}$ to allow identification of asteroids in the images; over this time period asteroids will typically move $0.3^{\prime}-2^{\prime}$. Because of Serpens's relatively large ecliptic latitude, $\sim 24^{\circ}$, only a very small number

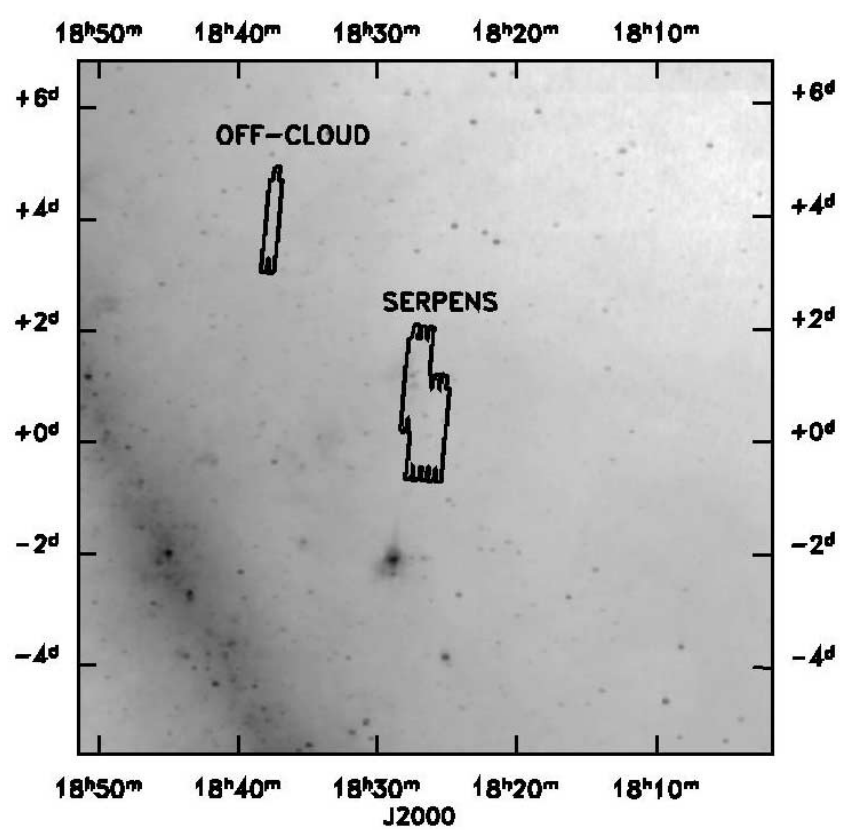

FIG. 1.-IRAS $25 \mu \mathrm{m}$ map showing the observed c2d regions in the Serpens cloud, both the star-forming region marked "SERPENS" and the low-extinction "OFF-CLOUD" area.
TABLE 2

Serpens Cloud Sky Coverage

\begin{tabular}{|c|c|c|c|}
\hline Region & $\begin{array}{l}24 \mu \mathrm{m} \\
\left(\operatorname{deg}^{2}\right)\end{array}$ & $\begin{array}{l}70 \mu \mathrm{m} \\
\left(\operatorname{deg}^{2}\right)\end{array}$ & $\begin{array}{c}160 \mu \mathrm{m} \\
\left(\mathrm{deg}^{2}\right)\end{array}$ \\
\hline 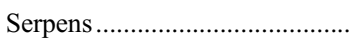 & 1.81 & 1.57 & 1.49 \\
\hline Off-cloud & 0.47 & 0.36 & 0.41 \\
\hline
\end{tabular}

of asteroids were seen, all of which were removed by requiring two-epoch detection in our final source lists. Typical integration times are $30 \mathrm{~s}$ at $24 \mu \mathrm{m}, 15 \mathrm{~s}$ at $70 \mu \mathrm{m}$, and $3 \mathrm{~s}$ at $160 \mu \mathrm{m}$. Additional GTO observations east of the region of highest emission are not included in this analysis because a different observing strategy was used. Those observations could, however, be added to ours in order to construct a somewhat larger mosaic of the region.

Figure 2 shows the three individual images produced for the MIPS bands, as well as a false-color image of the three together. Harvey et al. (2007) show an additional image combining the $24 \mu \mathrm{m}$ data with IRAC observations, as well as enlargements of the two main clusters observed. Note that unlike the IRAC instrument, the three wavelengths of MIPS all have diffraction-limited spatial resolution, which means that the resolution varies dramatically between $24 \mu \mathrm{m}\left(\sim 6^{\prime \prime}\right)$ and $160 \mu \mathrm{m}\left(\sim 40^{\prime \prime}\right)$.

Our data reduction is described in detail by Evans et al. (2007), but we summarize the important details here. In addition, previous versions of the $\mathrm{c} 2 \mathrm{~d}$ pipeline, some of which still apply to these data, have been described in more detail by Rebull et al. (2007) and Young et al. (2005). We began our data reduction with the BCD images, processed in this case by the standard Spitzer Science Center (SSC) S13.2 pipeline. Following this the three MIPS channels underwent slightly different processing paths in our $\mathrm{c} 2 \mathrm{~d}$ reduction. The $24 \mu \mathrm{m}$ data were mosaicked with the SSC's MOPEX software (Makovoz \& Marleau 2005) after processing in the $\mathrm{c} 2 \mathrm{~d}$ pipeline to reduce artifacts, e.g. " "jailbars" near bright sources. Point sources were extracted with "c2dphot" (P. M. Harvey et al. 2007, in preparation), a source extractor based on "DoPHOT" (Schechter et al. 1993), which utilizes the mosaics for source identification but the stack of individual BCDs for each identified object to provide the photometry and position information. We have estimated our completeness limit at $24 \mu \mathrm{m}$ in a manner similar to that described for our IRAC photometry (Harvey et al. 2006). We inserted a number of artificial sources into the $24 \mu \mathrm{m}$ mosaic at random positions over a range of brightness covering the range $2 \mathrm{mag}<[24]<12 \mathrm{mag}$ and then tested whether they were properly extracted. We also produced a mosaic with only artificial sources (no real ones) but a noise level comparable to that in the observed image, and we tested the completeness of extraction from that artificial image to estimate the effects of confusion in this relatively high source density region. Figure 3 shows the results from these tests. Clearly at the fainter flux levels, the effects of high source density are important to the true completeness level in Serpens, e.g., $[24]>9.5 \mathrm{mag}$.

The processing of the $70 \mu \mathrm{m}$ data followed a path similar to that at $24 \mu \mathrm{m}$ with two exceptions. At $70 \mu \mathrm{m}$ the SSC produces two sets of BCDs, one of which is simply calibrated and another that is filtered spatially and temporally in a manner that makes point-source identification easier but does not conserve flux for brighter sources or for diffuse emission. We produced mosaics of both the unfiltered and the filtered products using MOPEX 


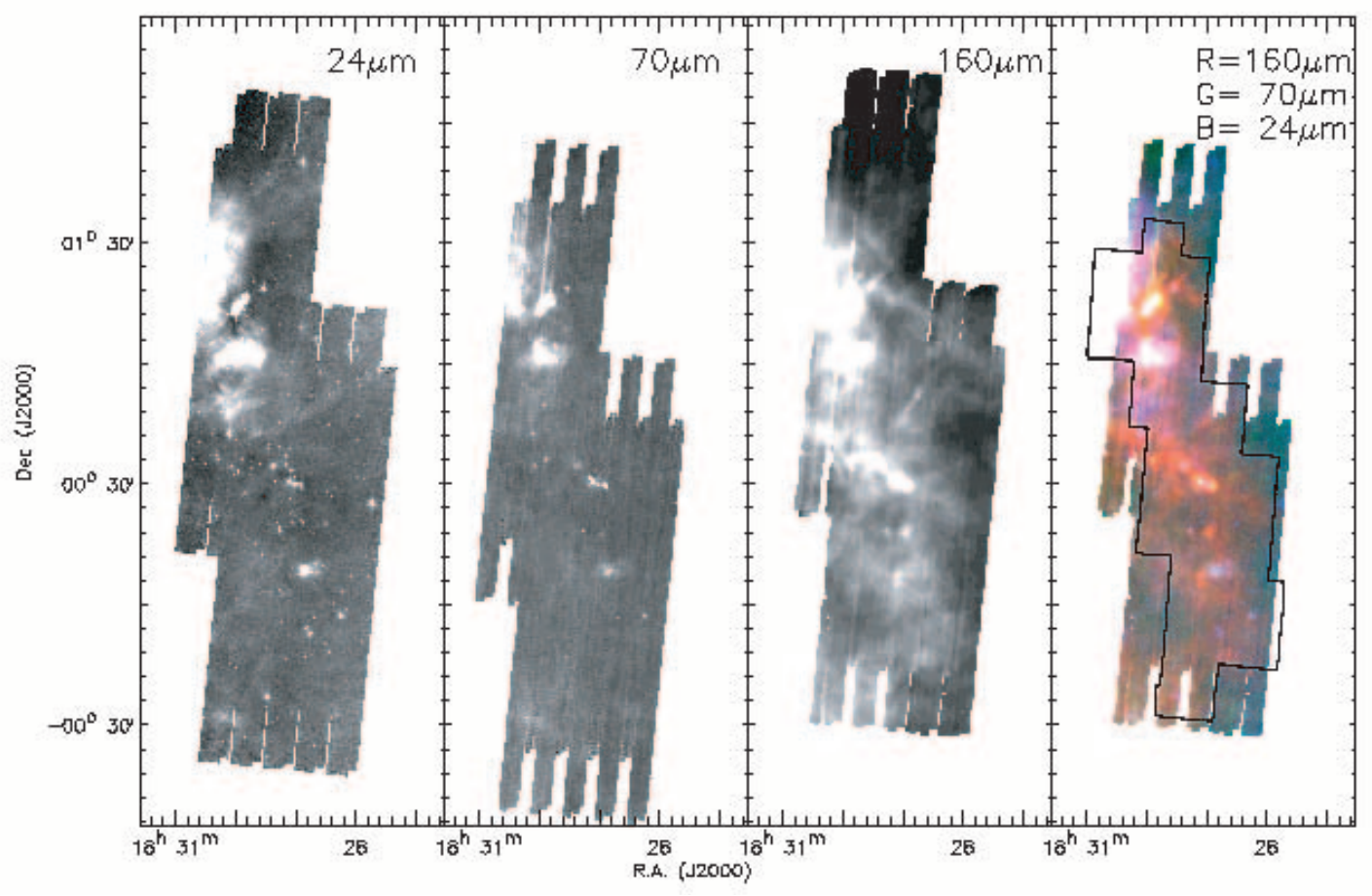

FIG. 2.-Registered Serpens 24, 70, and $160 \mu$ m images of the c2d MIPS region. The color image is a composite of all three bands and includes only the 1.27 deg ${ }^{2}$ area where data are available for each of the three bands. Colors represent $160 \mu \mathrm{m}$ (red), $70 \mu \mathrm{m}$ ( green), and $24 \mu \mathrm{m}$ (blue). The black outline shows the region where four bands of IRAC data were observed (Harvey et al. 2006).

on the native BCD pixel scale. Point sources were extracted using APEX (Makovoz \& Marleau 2005). Source reality was checked by hand inspection and comparison with the $24 \mu \mathrm{m}$ source list. Generally, the filtered mosaics were used for pointsource extraction, but above $F(70) \sim 2 \mathrm{Jy}$, we used the unfiltered data. Above $F(70) \sim 23 \mathrm{Jy}$, sources begin to be saturated. At these very high flux levels we used a procedure to fit the wings of the source profile; these data have been assigned a

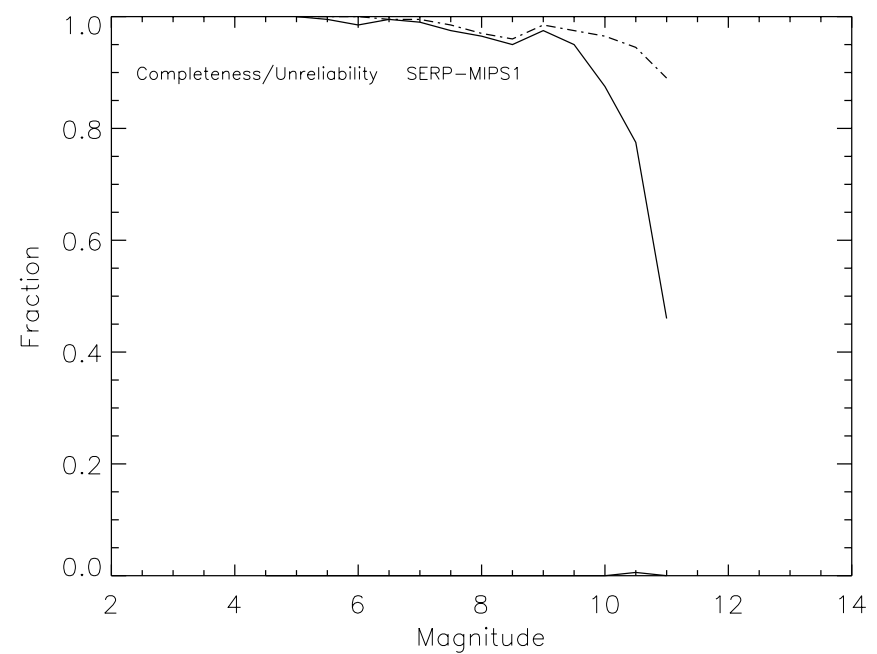

FIG. 3.-Completeness test at $24 \mu \mathrm{m}$. The upper solid line shows the measured completeness fraction for artificial sources inserted into the observed $24 \mu \mathrm{m}$ mosaic image of Serpens as a function of magnitude. The slightly higher dotdashed line shows the completeness fraction for sources inserted into an artificial image with no real sources but with a noise level equal to that in the observed data. The lower solid line (mostly equal to zero) shows the fraction of "unreliable" sources, i.e., sources extracted that were not real. higher uncertainty because of the inherent uncertainties in this procedure.

Complete tables of source positions and flux densities for likely cloud members in Serpens are given by Harvey et al. (2007) for our 3.6-70 $\mu \mathrm{m}$ observations. At $160 \mu \mathrm{m}$ our processing was limited to producing a native pixel scale mosaic using interpolation to fill in missing pixels and point-source extraction from the unfiltered mosaic. We extracted four nominal point sources in the entire mapped area. Two of these are associated with obvious multiple clumps of $24 \mu \mathrm{m} / 70 \mu \mathrm{m}$ sources. The other two, SSTc2d J1829167+0018225 (associated with IRAS 18267+0016) and SSTc2d J18293197+0118429 (associated with source 159 of Kaas et al. 2004), are likely powered mostly by single, shorter wavelength sources. Table 3 lists the positions and flux densities of these four nominal point sources with short comments, since their $160 \mu \mathrm{m}$ photometry is not described in any of our other publications on Serpens. None of these are in the core area of either of the main clusters. This is because large areas in those clusters are saturated, and the close spacing of many bright sources leads to the complicated, extended structure seen in Figure 2 at $160 \mu \mathrm{m}$, without obvious pointlike sources.

After extraction, the source lists were band merged with our IRAC source lists for Serpens (Harvey et al. 2006) and the 2MASS catalog of $J, H$, and $K_{s}$ photometry (Skrutskie et al. 2006) as described by Evans et al. (2007). The radius for source matching with shorter wavelength detections was $4^{\prime \prime}$ at $24 \mu \mathrm{m}$ and $8^{\prime \prime}$ at $70 \mu \mathrm{m}$. Table 4 lists the number of sources extracted at 24 and $70 \mu \mathrm{m}$ and some examples of statistics of numbers identified with shorter wavelength sources. In addition to band merging, sources undergo a classification process based on the available photometry, 2MASS, IRAC, and MIPS. For the purposes of this paper the most important classification is that 
TABLE 3

$160 \mu \mathrm{m}$ Point Sources

\begin{tabular}{|c|c|c|c|c|}
\hline $\begin{array}{c}\text { R.A. } \\
(\mathrm{J} 2000.0)\end{array}$ & $\begin{array}{c}\text { Decl. } \\
(\mathrm{J} 2000.0)\end{array}$ & $\begin{array}{l}\text { Flux } \\
(\mathrm{mJy})\end{array}$ & Comment & YSO Number $^{a}$ \\
\hline $182932.3 \ldots \ldots \ldots \ldots \ldots \ldots .$. & +011856 & 24000 & Single $24 \mu \mathrm{m} / 70 \mu \mathrm{m}$ source & 104 \\
\hline 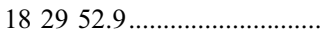 & +003609 & 18200 & Cluster of four $24 \mu \mathrm{m}$ sources & $\ldots$ \\
\hline 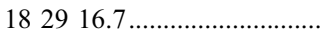 & +001820 & 10000 & Single $24 \mu \mathrm{m} / 70 \mu \mathrm{m}$ source & 88 \\
\hline $1828 \quad 15.7 \ldots \ldots \ldots \ldots \ldots \ldots \ldots \ldots$ & -000311 & 6070 & Cluster of four $24 \mu \mathrm{m}$ sources & $\ldots$ \\
\hline
\end{tabular}

Noте.-Units of right ascension are hours, minutes, and seconds, and units of declination are degrees, arcminutes, and arcseconds.

${ }^{\mathrm{a}}$ YSO number from Harvey et al. (2007).

of "star," which implies a spectral energy distribution (SED) that is well fitted as a reddened stellar photosphere without requiring any excess infrared emission from possible circumstellar dust. The data reported here consist of a subset of all the sources extracted in Serpens. The entire catalog is available from the SSC Web site..$^{10}$ For this paper we have limited our discussion to sources with a signal-to-noise ratio $(\mathrm{S} / \mathrm{N})$ greater than 5 and to sources found in both epochs of observation to eliminate asteroids. These limits lead to a very high reliability for the objects reported here, probably greater than $98 \%$.

In addition to our reduction of the Serpens cloud and offcloud data, we have also processed a $5.3 \mathrm{deg}^{2}$ portion of the SWIRE Spitzer Legacy data (Surace et al. $2004^{11}$ ) from the ELAIS N1 field through our c2d pipeline. Since this field is almost entirely populated by Galactic stars and extragalactic objects, it provides an additional control field against which to compare our Serpens cloud population as discussed below. Note that the SWIRE observations go approximately a factor of 4 deeper than $\mathrm{c} 2 \mathrm{~d}$ due to increased integration time.

\section{RESULTS}

\subsection{Extended Emission}

The $160 \mu \mathrm{m}$ emission traces the coolest and most extended dust seen with MIPS. Figure 4 shows an image of the $160 \mu \mathrm{m}$ emission together with contours of the optical extinction. Also shown are the locations of the two main clusters of young stellar objects in Serpens: the core cluster A, and cluster B (also called the G3-G6 cluster by Djupvik et al. 2006). The optical

10 See http://ssc.spitzer.caltech.edu/legacy/all.html.

11 Available at http://ssc.spitzer.caltech.edu/legacy/.

TABLE 4

Serpens Cloud Detection Statistics

\begin{tabular}{|c|c|}
\hline Wavelength(s) & Source Number \\
\hline 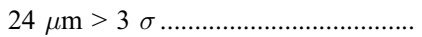 & 2635 \\
\hline 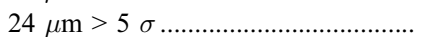 & 1494 \\
\hline 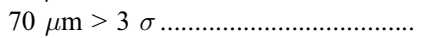 & 97 \\
\hline 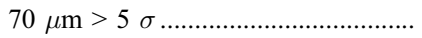 & 88 \\
\hline 24 and $70 \mu \mathrm{m}>5 \sigma \ldots \ldots \ldots \ldots \ldots \ldots \ldots \ldots$ & 75 \\
\hline $24 \mu \mathrm{m}$ and $2 \mathrm{MASS} K_{s}>5 \sigma \ldots \ldots \ldots$ & 1085 \\
\hline 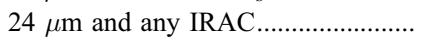 & $1040^{\mathrm{a}}$ \\
\hline 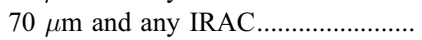 & 77 \\
\hline
\end{tabular}

a The greater number of matches between $24 \mu \mathrm{m}$ and $K_{s}$ vs. IRAC is due to the smaller area coverage of the IRAC data.

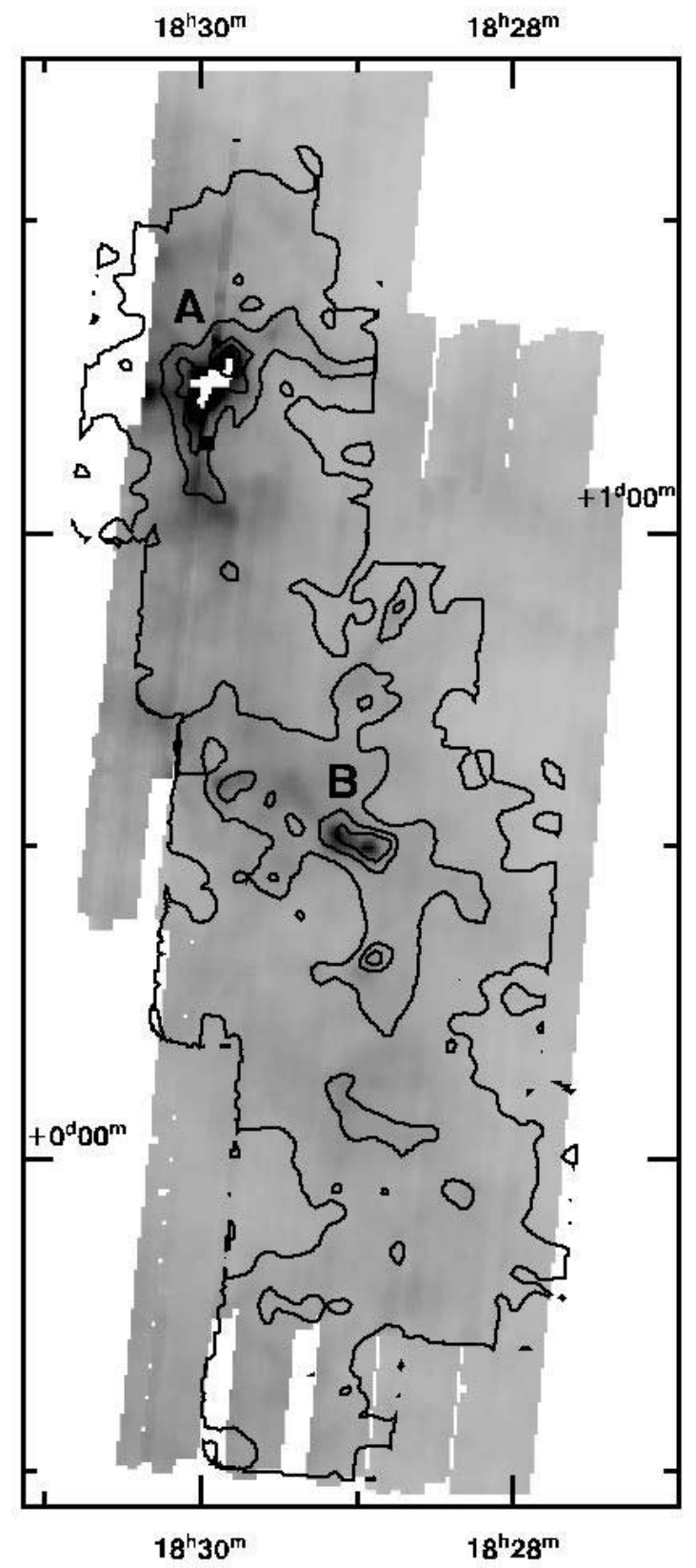

FIG. 4.-Contours of $A_{v}$ at levels of 5, 10, 20, and 30 mag determined from 2MASS and Spitzer c2d IRAC data overlaid on the Serpens $160 \mu$ m image. The visual extinction and $160 \mu \mathrm{m}$ emission are quite well correlated. The locations of clusters $\mathrm{A}$ and $\mathrm{B}$ are indicated. 


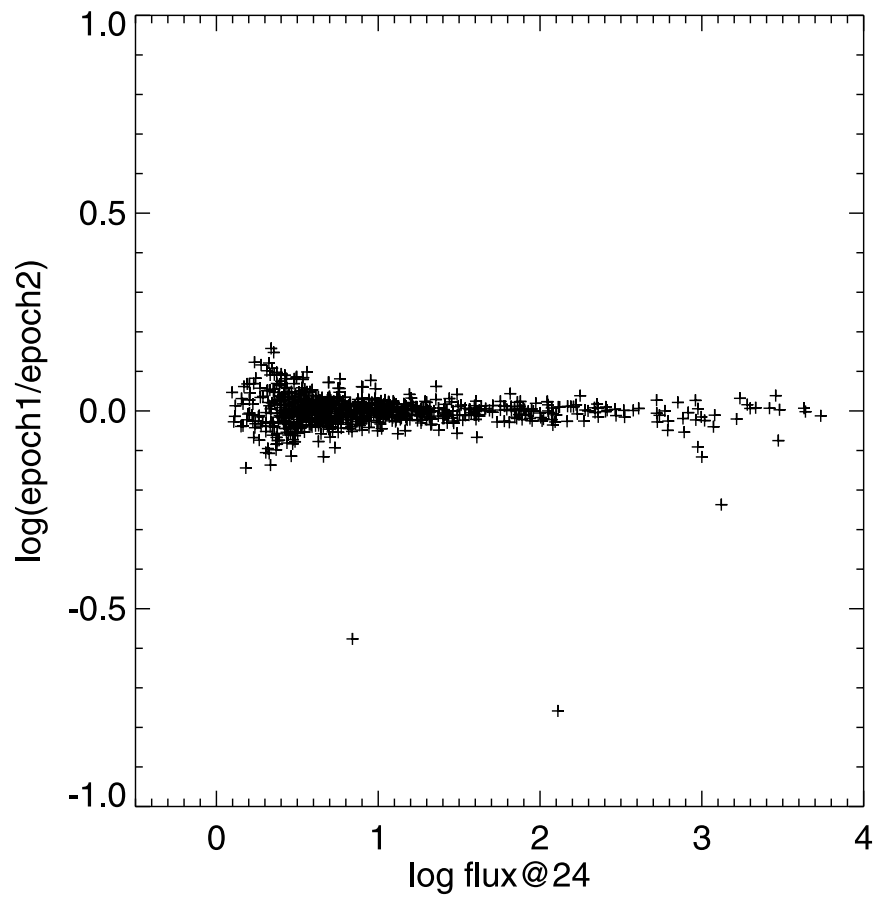

FIG. 5.- Search for time variability in the Serpens $24 \mu \mathrm{m}$ data; plot of $\log$ flux ratio of epoch 1 to epoch 2 vs. $\log$ flux density ( $\mathrm{mJy}$ ) for the combined epoch data. There is no verifiable time-variable source in the cloud based on these data.

extinction has been estimated by our fitting of the objects that were well characterized as extincted stellar photospheres. This figure shows a very close correlation between the coolest dust and the dust that is associated with optical extinction. The figure also clearly shows that the two high stellar density clusters, A and $\mathrm{B}$, are located in areas of maximum extinction, as we discuss further in $\S 3.5$.

\section{2. $24 \mu \mathrm{m}$ Time Variability}

Since many pre-main-sequence stars exhibit variable optical emission, we conducted a simple examination of the $24 \mu \mathrm{m}$ fluxes from the two observed epochs, similar to that in Perseus by Rebull et al. (2007) and for the IRAC data in Serpens (Harvey et al. 2007). As shown in Table 1, the time difference between the two epochs of observation was of order $4 \mathrm{hr}$. Figure 5 shows the ratio of the $24 \mu \mathrm{m}$ flux density between the two epochs for all the extracted sources whose $\mathrm{S} / \mathrm{N}$ was above 5 that were detected in both epochs of observation. Although there are a few outliers beyond the limits expected on the basis of the S/Ns, these are all readily explained as due to poor photometry near the edges of the mosaic or problems due to source confusion or adjacency to bright sources. This is consistent with the findings by Rebull et al. (2007) for the Perseus $24 \mu \mathrm{m}$ sources and by Harvey et al. (2007) for the Serpens IRAC sources. Although there are undoubtedly some variable sources in these clouds, the observing techniques of the $\mathrm{c} 2 \mathrm{~d}$ program were not designed to enable reliable detection of modestly variable objects.

\subsection{Source Counts}

Because the Serpens star-forming cloud is so close to the Galactic plane, $b \sim 5^{\circ}$, the vast majority of the sources detected

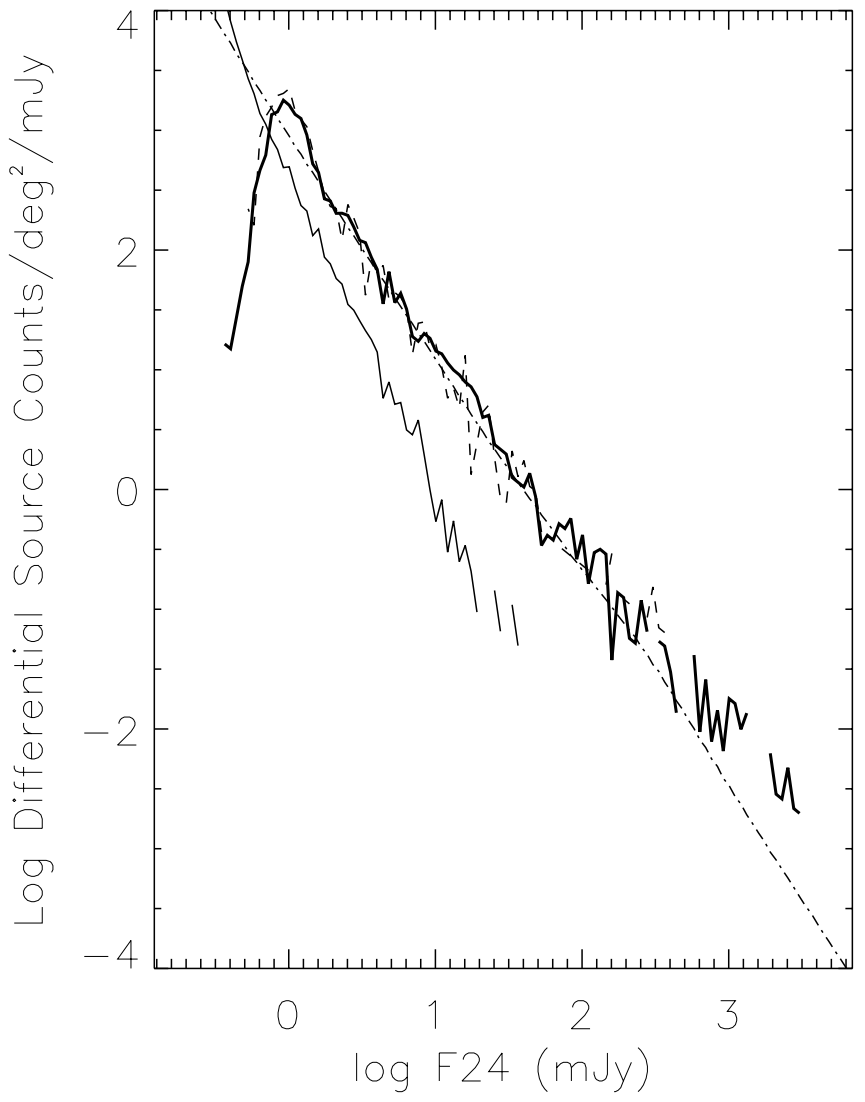

FIG. 6.-The $24 \mu \mathrm{m}$ source counts in the Serpens MIPS field (thick solid line) and off-cloud region (dashed line). SWIRE galaxy counts (thin solid line) fall below the Serpens data at our flux limit of $1 \mathrm{mJy}$. The predicted source counts from the Wainscoat model at $25 \mu \mathrm{m}$ (Wainscoat et al. 1992) are shown by the dotdashed line.

at the shorter wavelengths are background stars in the Galaxy. At fainter flux levels, background extragalactic objects constitute a significant population. In order to estimate the background Galactic star numbers, we have used the Wainscoat et al. (1992) model provided by J. Carpenter (2001, private communication). Figure 6 shows the predicted star counts from the model together with the observed counts at $24 \mu \mathrm{m}$ for both the Serpens cloud and the off-cloud region. Also shown in the figure are the source counts from the c2d-processed SWIRE ELAIS N1 field, which are largely extragalactic for fluxes below a few mJy. This figure shows that contamination by Galactic stars at the brighter fluxes and by extragalactic sources at the faint end is a significant problem for identifying Serpens cloud members. To address this problem, we discuss our use of several color and flux criteria in the following section. It is also apparent that there is an excess of bright $(F>300 \mathrm{mJy})$ sources relative to the expected background counts. This excess is, in fact, real and represents the bright end of the YSO candidate population discussed in the following section.

\subsection{Color-Magnitude Diagrams}

The $\mathrm{c} 2 \mathrm{~d}$ team has discussed in a number of studies how the use of color-magnitude and color-color diagrams can separate likely young cloud members with infrared excesses from reddened stars and many background extragalactic sources (Young et al. 2005; Harvey et al. 2006, 2007; Rebull et al. 2007). Since 


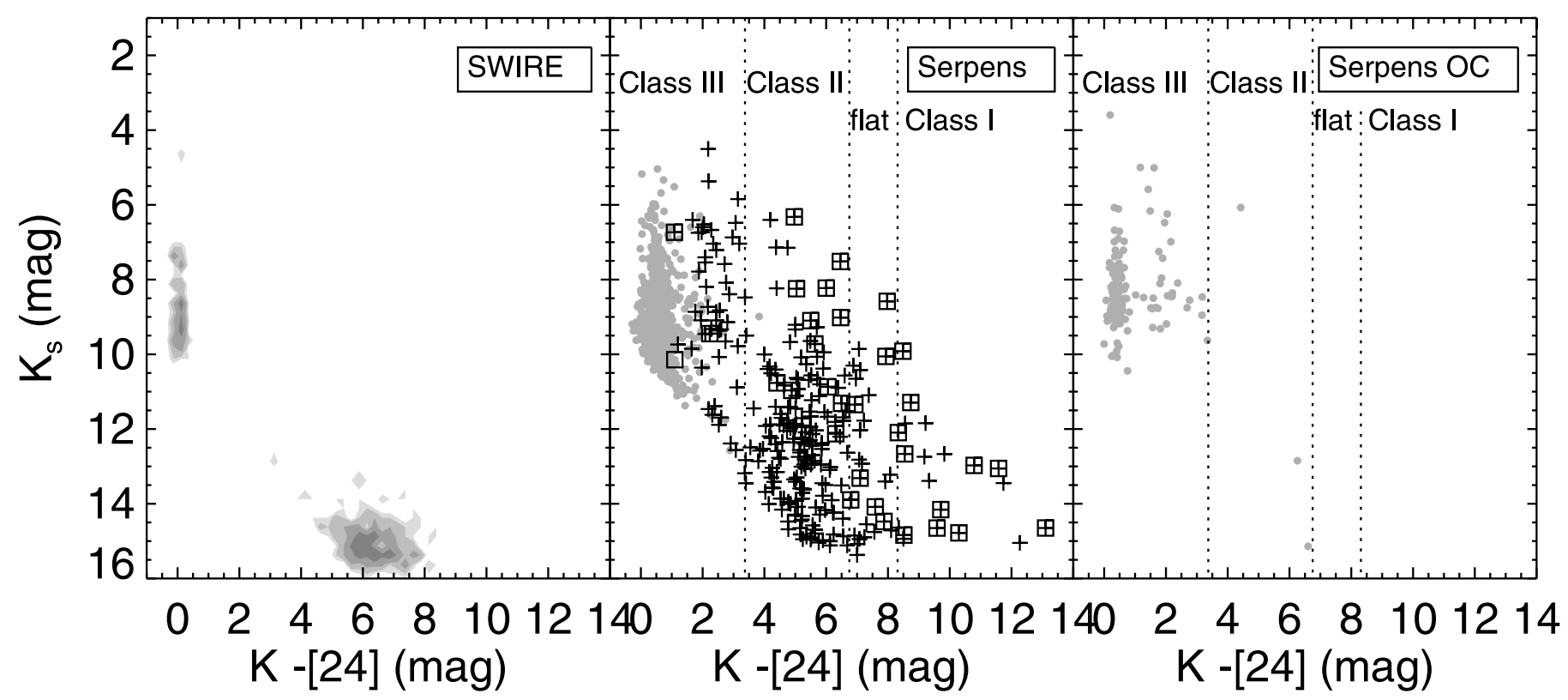

FIG. 7.-Color-magnitude diagram for $K_{s}$ vs. $K_{s}-[24]$ for objects in SWIRE (left), the Serpens cloud (middle), and the Serpens off-cloud region (right). The SWIRE counts are shown as a surface density, with darker implying higher density. Objects in SWIRE are expected to be mostly galaxies (objects with $K_{s} \gtrsim 14$ ) or stellar photospheres (objects with $K_{s}-[24] \lesssim 1$ ). For the Serpens cloud and off-cloud plots, filled gray circles represent objects with SEDs resembling photospheres, and plus signs represent the remaining objects. An additional box around a point denotes that it was also detected at $70 \mu \mathrm{m}$. Objects that are candidate young objects have colors unlike those objects found in SWIRE, e.g., $K_{s} \lesssim 14$ and $K_{s}-[24] \gtrsim 1$. Dashed lines denote the divisions between Class I, flat, Class II, and Class III objects; to omit foreground and background stars, we have further imposed a $K_{s}-[24]>2$ requirement on our Class III objects (see text).

TABLE 5

Classification Based on $K_{s}-[24]$

\begin{tabular}{|c|c|}
\hline Classification & Serpens Source Count ${ }^{\mathrm{a}}$ \\
\hline Number with $K_{s}-[24]>2, K_{s}<14 \ldots$ & 250 \\
\hline Number with $K_{s}-[24]>2, K_{s}<14$, and Class I $K_{s}-[24]$ color $\ldots \ldots \ldots$. & $15(6 \%)$ \\
\hline Number with $K_{s}-[24]>2, K_{s}<14$, and "flat" $K_{s}-[24]$ color .......... & $21(8 \%)$ \\
\hline Number with $K_{s}-[24]>2, K_{s}<14$, and Class II $K_{s}-[24]$ color $\ldots \ldots . .$. & $158(63 \%)$ \\
\hline Number with $K_{s}-[24]>2, K_{s}<14$, and Class III $K_{s}-[24]$ color...... & $56(22 \%)$ \\
\hline
\end{tabular}

${ }^{\text {a }}$ Since a 2MASS detection is required to be included in these statistics, very cold or deeply embedded sources are not present in these counts, e.g., those sources in Table 6 . 


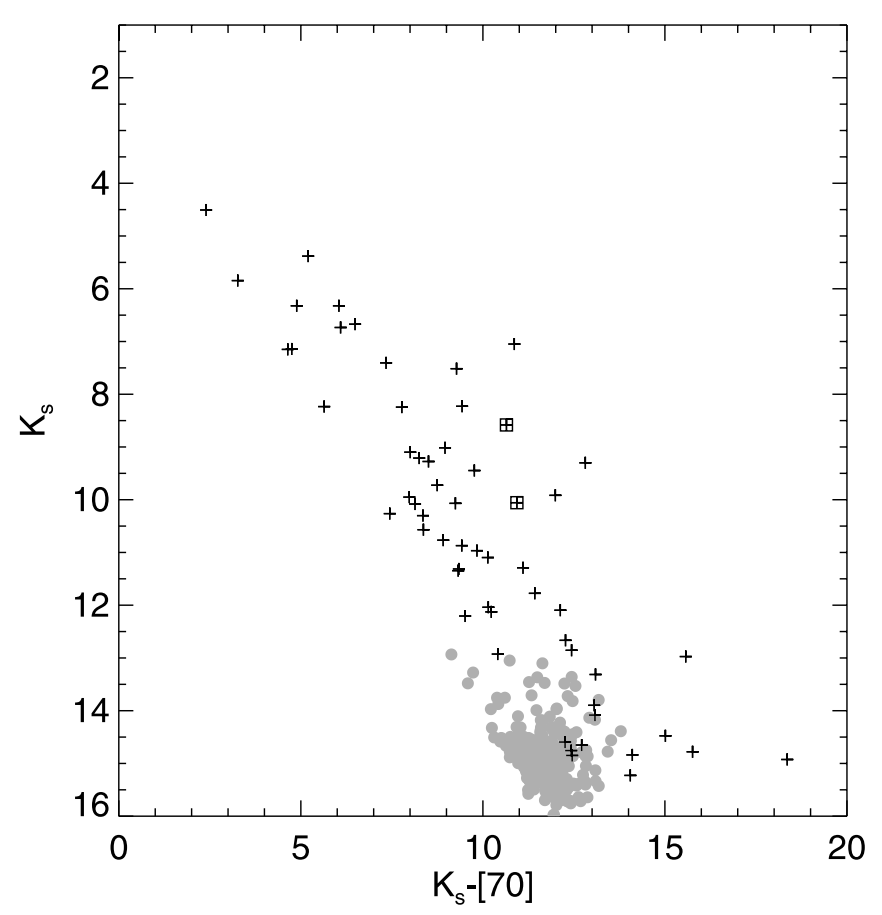

Fig. 8.-Color-magnitude diagram of $K_{s}$ vs. $K_{s}-[70]$ for Serpens (plus signs) with data from the full SWIRE survey (gray filled circles) included for comparison.

nearly half of the area covered by our MIPS $24 \mu \mathrm{m}$ observations was not observed with IRAC (Harvey et al. 2006), we utilize the color and magnitude criteria developed by Young et al. (2005) and refined by Rebull et al. (2007) and Chapman et al. (2007) to isolate a candidate YSO population without requiring the existence of IRAC data. The most populated diagram is naturally the color-magnitude diagram of $K_{s}$ versus $K_{s}-[24]$ because of the much larger number of $24 \mu \mathrm{m}$ sources than $70 \mu \mathrm{m}$ ones. Figure 7 shows the distribution of sources in this diagram for the 1453 sources with $\mathrm{S} / \mathrm{N}$ above 5 at $24 \mu \mathrm{m}$ and with 2MASS $K_{s}$ matches within $4^{\prime \prime}$. This distribution is very similar to that seen in other well-populated c2d clouds such as Perseus (Rebull et al. 2007). A comparison of the SWIRE results, the Serpens off-cloud results, and the Serpens cloud data shows that (1) objects in our "star" class fall in a relatively narrow band with blue $K_{s}-[24]$ colors $\left(K_{s}-[24]<1\right)$, as would be expected, and (2) the part of the diagram toward redder colors is populated by a number of sources in Serpens that are not seen in either the off-cloud region or the SWIRE data set, except at $K$ magnitudes fainter than $K_{s} \sim 14$. This allows us to assign a high probability that sources in the region $K_{s}<14$ and $K_{s}-[24]>2$ are Serpens cloud YSO candidates with excess emission at $24 \mu \mathrm{m}$ probably due to circumstellar dust. Note that the off-cloud area does have a population of moderately reddened objects $\left(K_{s}-[24]<2\right)$, well fitted as stellar photospheres that are not seen in the SWIRE sample, simply because even the off-cloud area has more reddening than the high Galactic latitude ELAIS N1 region. In order to categorize our YSO candidates crudely in terms of evolutionary state, we have drawn lines in Figure 7 indicating where objects would fall based on the YSO source classification criteria of Greene et al. (1994) using the $K_{s}-[24]$ color to measure the spectral slope. Table 5 lists the number of candidates and the number in each of the four classes. Although asymptotic giant branch (AGB) stars

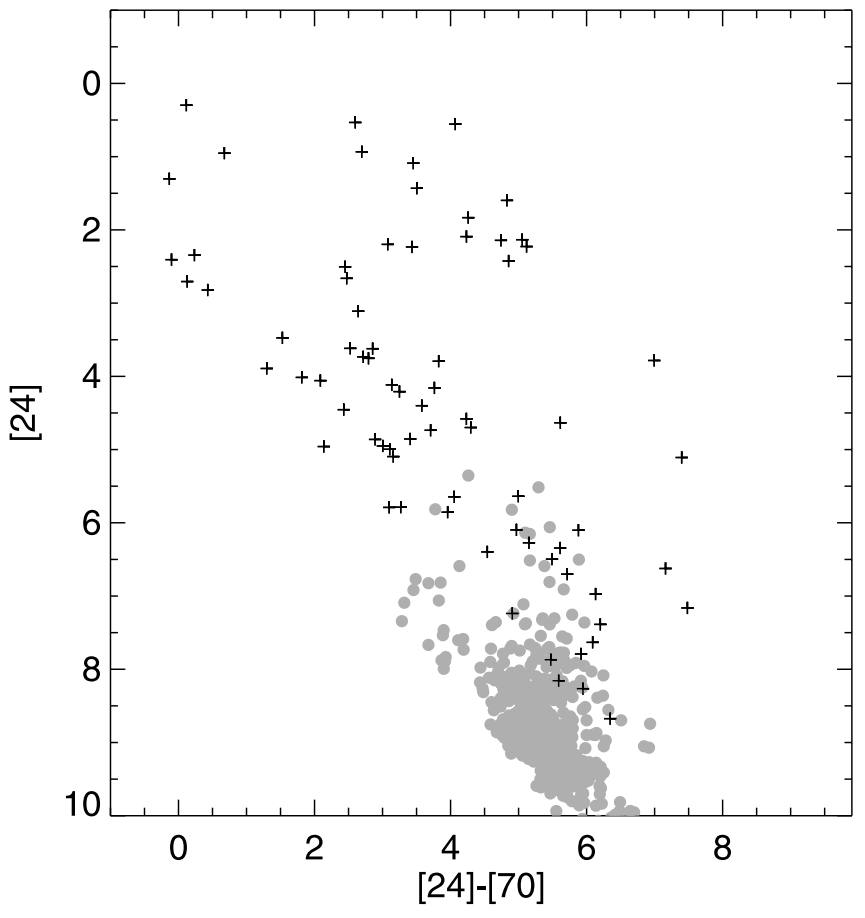

FIG. 9.-Color-magnitude diagram of [24] vs. [24] - [70] for Serpens ( plus signs) with data from the full SWIRE survey (gray filled circles) included for comparison.

with substantial mass loss also exhibit mid-infrared excesses, Harvey et al. (2006) have argued that the number expected in this area is less than or of order a half-dozen (four of which have already been confirmed spectroscopically as AGB interlopers by B. Merín et al. 2007, in preparation). The positions of and photometry for the YSO candidates that are not in the area covered by IRAC are given by Harvey et al. (2007), along with those in the IRAC area.

Harvey et al. (2007) discuss the comparison between YSOs selected by the criteria used here ( $K_{s}$ and $24 \mu \mathrm{m}$ data only) and the more restrictive criteria possible with the combination of IRAC data. They basically find that we actually may have missed eight or nine YSOs in the area not covered by IRAC and included a very few, three or four, that may be background extragalactic sources. But the overall conclusion is that there is a good correspondence between the YSO candidates found using only MIPS and 2MASS and those selected with a more complete data set. It is also clear that the area mapped by both IRAC and MIPS, $0.85 \mathrm{deg}^{2}$, contains a much higher density of YSOs, 235 or $276 \mathrm{deg}^{-2}$, than does the area only covered by MIPS $/ 24 \mu \mathrm{m}$, with 51 YSOs or $54 \mathrm{deg}^{-2}$. Even if we exclude the area of the two high-density clusters, the area covered by the combined IRAC/MIPS observations has a YSO density a factor of 4 higher than the area not included in the IRAC observations.

We have also plotted our photometry in two other color/ magnitude spaces for comparison with other c2d clouds. Figure 8 shows the distribution of sources in $K_{s}$ versus $K_{s}-[70]$ space. As observed by Rebull et al. (2007) in Perseus, there are a large number of likely cloud members at much brighter $K_{s}$ magnitudes than seen for SWIRE extragalactic objects. In addition, there is a small population of faint (in $K_{s}$ ) objects that are redder than any of the SWIRE objects in both Serpens and Perseus. The four objects redder than $K_{s}-[70]=15$ are 
TABLE 6

The Most Embedded OвJects

\begin{tabular}{|c|c|c|c|c|c|c|c|c|}
\hline Name/Position & YSO Number ${ }^{\mathrm{a}}$ & $\begin{array}{c}3.6 \mu \mathrm{m} \\
(\mathrm{mJy})\end{array}$ & $\begin{array}{c}4.5 \mu \mathrm{m} \\
(\mathrm{mJy})\end{array}$ & $\begin{array}{c}5.8 \mu \mathrm{m} \\
(\mathrm{mJy})\end{array}$ & $\begin{array}{c}8.0 \mu \mathrm{m} \\
(\mathrm{mJy})\end{array}$ & $\begin{array}{c}24.0 \mu \mathrm{m} \\
(\mathrm{mJy})\end{array}$ & $\begin{array}{c}70.0 \mu \mathrm{m} \\
(\mathrm{mJy})\end{array}$ & Associated Source ${ }^{\mathrm{b}}$ \\
\hline SSTc2d J18285404+0029299.. & 40 & $5.81 \pm 0.50$ & $27.6 \pm 2.3$ & $44.8 \pm 2.6$ & $56.4 \pm 3.2$ & $918 \pm 85$ & $11100 \pm 1040$ & D62/D66 \\
\hline SSTc2d J18290619+0030432 …....... & 67 & $8.05 \pm 0.41$ & $45.0 \pm 2.8$ & $93.9 \pm 4.8$ & $129 \pm 7$ & $1320 \pm 139$ & $7240 \pm 713$ & $\mathrm{D} 90$ \\
\hline SSTc2d J18290675+0030343 …....... & 68 & $3.27 \pm 0.21$ & $11.7 \pm 0.7$ & $14.9 \pm 0.8$ & $20.7 \pm 1.2$ & $1000 \pm 105$ & $11400 \pm 1180$ & D94 \\
\hline SSTc2d J18290906+0031323 ........... & $\ldots$ & $<0.12$ & $0.29 \pm 0.03$ & $0.40 \pm 0.09$ & $0.31 \pm 0.08$ & $64.6 \pm 6.0$ & $6380 \pm 611$ & D101 \\
\hline SSTc2d J18294810+0116449 ........... & 135 & $1.96 \pm 0.10$ & $6.98 \pm 0.42$ & $12.1 \pm 0.6$ & $16.7 \pm 0.8$ & $219 \pm 21$ & $14900 \pm 1420$ & K241, SMM 9 \\
\hline SSTc2d J18295285+0114560 ........... & 155 & $8.65 \pm 0.44$ & $34.6 \pm 1.8$ & $72.0 \pm 3.4$ & $110 \pm 5$ & $1040 \pm 96$ & $5570 \pm 523$ & K276 \\
\hline SSTc2d J18295927+0114016 ........... & 195 & $2.72 \pm 0.28$ & $5.76 \pm 0.44$ & $7.78 \pm 1.16$ & $36.0 \pm 5.4$ & $109 \pm 19$ & $12200 \pm 1160$ & SMM 3 \\
\hline SSTc2d J18295992+0113116 _.......... & 198 & $2.77 \pm 0.16$ & $29.5 \pm 1.5$ & $103 \pm 4$ & $199 \pm 10$ & $2620 \pm 249$ & $6830 \pm 675$ & K331 \\
\hline
\end{tabular}

a Identifying number from YSO table in Harvey et al. (2007).

b D: Djupvik et al. 2006; K: Kaas et al. 2004; SMM: Davis et al. (1999).

all likely to be slightly less extreme versions of the sources discussed in the next section. Two of these are located in cluster A but tend to be around the outside of the tight cluster of very red objects. The other two are in a small grouping associated with the second of the four $160 \mu \mathrm{m}$ point sources listed in Table 3. Since all of these objects were also observed in our program with IRAC, they are also listed in the appropriate tables of Harvey et al. (2007), and all are considered high-probability YSOs.

The final color-magnitude diagram, [24] versus [24] - [70], is shown in Figure 9. Again this distribution is qualitatively similar to that in Perseus, although we find many fewer sources in the area overlapping the red edge of the extragalactic distribution than did Rebull et al. (2007) for their "rest of the cloud." The Serpens distribution is qualitatively more similar to that for the NGC 1333 portion of Perseus. Since many of the sources represented in this diagram for Serpens are located in one of the two principal clusters, A and B, in Serpens, it is perhaps not surprising that they would mimic some of the properties of similar young clusters like NGC 1333.

\subsection{The Most Embedded Objects}

We have selected the coldest, most obscured sources from our sample by looking for objects not detected in 2MASS but

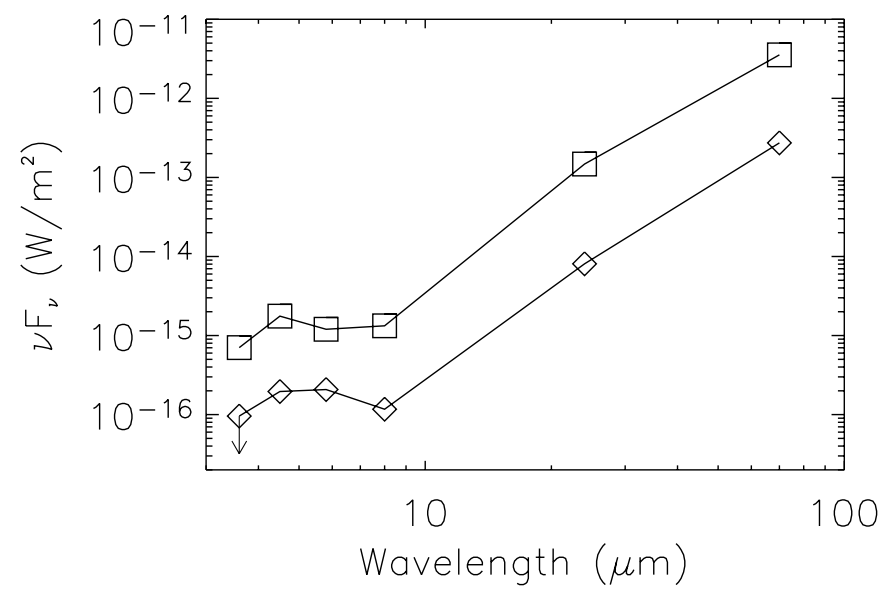

FIG. 10.-SED for the two most embedded sources in Table 6, one in cluster A (open squares; SSTc2d J1829463+0115219) and one in cluster B (open diamonds; SSTc2d J18290906+0031323, source "C" in Table 7), both of which appear to be associated with outflows. detected with reasonable $\mathrm{S} / \mathrm{N}$ at both 24 and $70 \mu \mathrm{m}$. There are 11 such objects in our surveyed area, and these are listed in Table 6. Interestingly, all 11 are located in the heart of either cluster A or B. In addition, as shown in Table 6 , all were detected in some or all IRAC bands. Their energy distributions are all consistent with a designation of Class I even though they are not included in Figure 7 since they were not detected in 2MASS. In fact, several of these objects are strongly enough peaked in the far-infrared that they have energy distributions consistent with some nominal Class 0 sources despite the fact that all were detected with IRAC. The class status of these will be discussed further using millimeter data by M. L. Enoch et al. (2007, in preparation). Figure 10 shows the SEDs for the two most embedded objects from Table 6 . Each of these appears to be associated with an outflow in its respective cluster, and both have very similar SEDs that differ only in their absolute flux level by a factor of $\sim 10$.

Table 6 shows also that the most embedded object in cluster B (whose SED is shown in Fig. 10) was not selected as a YSO by Harvey et al. (2007). The reason is that the flux at $3.6 \mu \mathrm{m}$ was too faint to meet the selection criteria of that study. The area within $15^{\prime \prime}$ of that source contains two other extracted compact sources in the $\mathrm{c} 2 \mathrm{~d}$ data set. The positions and photometry for all three are shown in Table 7, and an image of the area is shown in Figure 11. Although the source density is quite high, the $70 \mu \mathrm{m}$ contours shown in the figure are clearly centered on the northernmost source, "C". Source " $B$ " is a slightly extended source that may represent a separate exciting object or may just be the location of the most visible jet emission that has been discussed briefly by Harvey et al. (2007) in this region. Source " $A$ " is a faint but very red object about 6 " to the west of source $\mathrm{C}$ and appears to be a pointlike object in the images.

Figure 4 shows clearly that clusters A and B are located in the highest extinction parts of the cloud. Therefore, the lack of detection of the objects in Table 6 at $1-2.3 \mu \mathrm{m}$ may be due at least partly to the extinction of the cloud material in which they are embedded, in addition to individual circumstellar material. Although the nominal extinction values in these areas range up to $A_{v} \sim 35-40$, the fact that these values result from smoothing over $90^{\prime \prime}$ of the stellar distribution means that they probably underestimate the extinction in the most extreme regions. This association of the coldest objects with the highest extinction regions is similar to the correlation seen by Enoch et al. 
TABLE 7

Sources Marked in Figure 11

\begin{tabular}{|c|c|c|c|c|c|c|c|}
\hline Marker & Name/Position & $\begin{array}{c}3.6 \mu \mathrm{m} \\
(\mathrm{mJy})\end{array}$ & $\begin{array}{c}4.5 \mu \mathrm{m} \\
(\mathrm{mJy})\end{array}$ & $\begin{array}{l}5.8 \mu \mathrm{m} \\
(\mathrm{mJy})\end{array}$ & $\begin{array}{l}8.0 \mu \mathrm{m} \\
(\mathrm{mJy})\end{array}$ & $\begin{array}{c}24.0 \mu \mathrm{m} \\
(\mathrm{mJy})\end{array}$ & $\begin{array}{c}70.0 \mu \mathrm{m} \\
(\mathrm{mJy})\end{array}$ \\
\hline $\mathrm{A}^{\mathrm{a}}$ & SSTc2d J18290904+0031280 & $0.95 \pm 0.11$ & $2.78 \pm 0.23$ & $2.92 \pm 0.24$ & $5.03 \pm 0.40$ & $14.0 \pm 1.9$ & . \\
\hline С & SSTc2d J18290906+0031323 & $<0.12$ & $0.29 \pm 0.03$ & $0.40 \pm 0.09$ & $0.31 \pm 0.08$ & $64.6 \pm 6.0$ & $6380 \pm 611$ \\
\hline
\end{tabular}

${ }^{\text {a }}$ This is YSO 75 in Harvey et al. (2007).

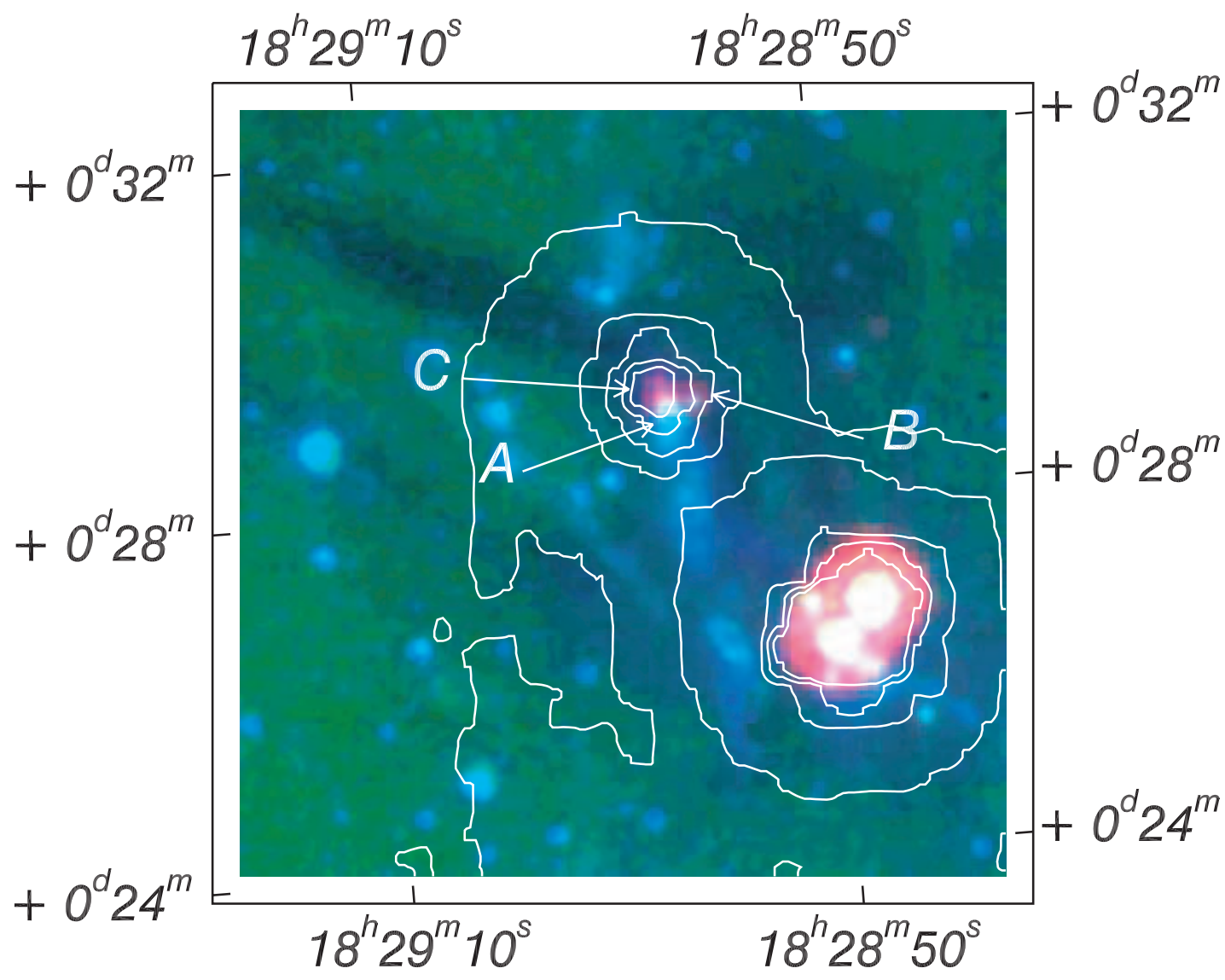

FIG. 11.-Three-color image of the eastern end of cluster B where the most embedded source, C, is located. This is the likely exciting source for an HH-like outflow visible in the IRAC data. The color scheme is blue, green, and red for 4.5, 8.0, and $24 \mu \mathrm{m}$, respectively. The contours of $70 \mu \mathrm{m}$ emission are also superimposed with levels at 40,80,160,240, and $320 \mathrm{MJy} \mathrm{sr}^{-1}$. Also shown are the positions of two other compact sources extracted from the images in this region. The letters correspond to positions/fluxes in Table 7 . 
(2006) between extinction and location of dense millimeter cores.

\section{SUMMARY}

We have described the basic observational characteristics of the $\mathrm{c} 2 \mathrm{~d}$ MIPS observations of the Serpens cloud. In a $1.5 \mathrm{deg}^{2}$ area we have found 250 YSO candidates on the basis of the $K_{s}-[24]$ color. An additional 11 objects can be identified on the basis of their 24 and $70 \mu \mathrm{m}$ fluxes and lack of detection by 2MASS. All of these YSO candidates will be discussed in more detail in a companion paper (Harvey et al. 2007). All of the most embedded objects are found in the central area of the two main clusters of YSOs previously identified in Serpens. The images and source catalogs derived from these data are all available on the SSC Web site (see footnote 10).

Support for this work, part of the Spitzer Legacy Science Program, was provided by NASA through contracts 1224608 , 1230782, and 1230779 issued by the Jet Propulsion Laboratory, California Institute of Technology, under NASA contract 1407. Astrochemistry in Leiden is supported by an NWO Spinoza grant and a NOVA grant. This publication makes use of data products from the Two Micron All Sky Survey, which is a joint project of the University of Massachusetts and the Infrared Processing and Analysis Center/California Institute of Technology, funded by NASA and the National Science Foundation. We also acknowledge extensive use of the SIMBAD database.
Cambrésy, L. 1999, A\&A, 345, 965

Chapman, N., et al. 2007, ApJ, in press

Davis, C. J., Matthews, H. E., Ray, T. P., Dent, W. R. F., \& Richer, J. S. 1999 , MNRAS, 309, 141

Djupvik, A. A., André, Ph., Bontemps, S., Motte, F., Olofsson, G., Galfalk, M., \& Floren, H.-G. 2006, A\&A, 458, 789

Enoch, M. L., et al. 2006, ApJ, 638, 293

Evans, N. J., II, et al. 2003, PASP, 115, 965

2007, Final Delivery of Data from the c2d Legacy Project: IRAC and MIPS (Pasadena: SSC)

Greene, T. P., Wilking, B. A., André, P., Young, E. T., \& Lada, C. J. 1994, ApJ, 434, 614

Harvey, P. M., et al. 2006, ApJ, 644, 307

Harvey, P. M., Merín, B., Huard, T. L., Rebull, L. M., Chapman, N., Evans, N. J., II, \& Myers, P. C. 2007, ApJ, 663, 1149
REFERENCES

Jorgensen, J. K., et al. 2006, ApJ, 645, 1246

Kaas, A. A., et al. 2004, A\&A, 421, 623

Makovoz, D., \& Marleau, F. R. 2005, PASP, 117, 1113

Padgett, D. L., et al. 2007, ApJ, in press

Porras, A., et al. 2007, ApJ, 656, 493

Rebull, L., et al. 2007, ApJ, in press

Schechter, P. L., Mateo, M., \& Saha, A. 1993, PASP, 105, 1342

Skrutskie, M., et al. 2006, AJ, 131, 1163

Straizys, V., Cernis, K., \& Bartasiute, S. 1996, Baltic Astron., 5, 125

Surace, J. A., et al. 2004, The SWIRE ELAIS N1 Image Atlases and Source Catalogs (Pasadena: SSC)

Wainscoat, R. J., et al. 1992, ApJS, 83, 111

Young, K. E., et al. 2005, ApJ, 628, 283

Zhang, C. Y., Laureijs, R. J., \& Clark, F. O. 1988a, A\&A, 196, 236

Zhang, C. Y., et al. 1988b, A\&A, 199, 170 